\title{
Identification of SNPs Affecting Porcine Carcass Weight with the 60K SNP Chip
}

\author{
Kwon Kang ${ }^{1,2}$, Dong-Won Seo ${ }^{1}$, Jae-Bong Lee ${ }^{3,4}$, Eun-Ji Jung ${ }^{3,4}$, Hee-Bok Park ${ }^{3,4}$, In-Cheol Cho ${ }^{5}$, Hyun-Tae Lim ${ }^{3}$ \\ and Jun Heon Lee ${ }^{1 *}$ \\ ${ }^{1}$ Department of Animal Science and Biotechnology, Chungnam National University, Daejeon 305-764, Korea, ${ }^{2}$ Korea genetic \\ station, Iksan, 570-952, Jeonbuk, Korea, ${ }^{3}$ Department of Animal Science, Gyeongsang National University, Jinju 660-701, \\ Korea, ${ }^{4}$ Institute of Agriculture and Life Science, Gyeongsang National University, Jinju 660-701, Korea, ${ }^{5}$ National \\ Institute of Animal Science, Rural Development Administration, Jeju 690-150, Korea
}

\begin{abstract}
Carcass weight $(\mathrm{CW})$ is one of the most important economic traits in pigs, directly affecting the income of farmers. In this study, a genome wide association study was performed to detect significant single nucleotide polymorphisms (SNPs) affecting CW in pigs derived from a $F_{2}$ intercross between Landrace and Korean native pig (KNP). Using high-density porcine SNP chips, highly significant SNPs were identified on SSC12. Two candidate genes, LOC100523510 and LOC100621652, were subsequently selected within this region and further investigated. Within these candidate genes, five SNPs were identified and genotyped using the VeraCode GoldenGate assay. The results revealed that one SNP in the LOC100621652 gene and four SNPs in the LOC100523510 gene are highly associated with CW. These SNP markers can thus have significant applications for improving CW in KNP. However, the functions of these candidate genes are not fully understood and require further study.
\end{abstract}

(Key words : Candidate gene, Carcass weight, Pig, SNP chip, SSC12)

\section{INTRODUCTION}

Quantitative trait loci (QTLs) refer to the genomic regions affecting quantitative traits that usually have continuous variables. These quantitative traits can be economically important to the livestock industry because of their impact on growth, meat quality, and even reproduction. Carcass weight $(\mathrm{CW})$ (the weight of the whole livestock body, except for the head, internal organs, feet and leather) is one of the most important quantitative economic traits and the increased yield of CW can directly affect the income of farms.

The identification of QTL regions can be performed through statistical modeling. Normally, the identification of QTL is performed through linkage analysis with microsatellite markers that cover the whole genome. However, this genome-wide linkage analysis has limitations when large distances exist between markers. However, the recent development of high throughput analysis using single nucleotide polymorphisms (SNPs) can enable genome-wide association studies (GWAS) that can efficiently identify QTL with smaller confidence intervals (Ernst and Steibel, 2013).

Until recently, thirty-five QTLs affecting $\mathrm{CW}$ were identified over nearly the whole genome in pigs. Six QTLs were identified in SSC1, eight in SSC4, three each in SSC7 and SSC8, and two each in SSC10 and SSC14. In addition, one QTL was identified in six chromosomes (SSC 2, 3, 5, 6, 12, 13). The $\mathrm{X}$ chromosome was also found to contain four CW QTLs (Animal QTLdb, http://www.animalgenome.org/cgibin/QTLdb/SS/index).

The Korean native pig (KNP), only found on Jeju Island in Korea, is highly adapted to the Korean environment, including climate. The KNP is one of the most important animal resources in Korea, and well-known for its great taste due to firm white fat and fine muscle fibers compared with other breeds (Jin et al., 2001; Kwon, 2006; Cho et al., 2007). On the other hand, the KNP has a low growth rate and small mature body weight compared to other breeds therefore, the overall profit from using this breed is not high (Cho et al., 2001). To overcome this weakness, the identification of markers in the KNP CW QTL region can greatly

* Corresponding author: Dr. Jun Heon Lee, Department of Animal Science and Biotechnology, College of Agriculture and Life Sciences, Chungnam National University, Daejeon 305-764, Republic of Korea. Tel: +82-42-821-5779, Fax: +82-42-8259754, E-mail:junheon@cnu.ac.kr 
help increase the growth and mature body weight of KNP

For the last 10 years, QTL researches and association studies using the KNP have focused on linkage maps using microsatellite markers and the construction of a BAC library for the identification of associations with economic traits (Jeon et al., 2003; Kim et al., 2004; Kim et al., 2007). However, the recent development of SNP chips enables GWAS analysis and the direct identification of linked SNPs (Balding, 2007). Examples of GWAS studies include those for backfat thickness and postmortem $\mathrm{pH}$ differences (Yoo et al., 2011), meat quality and biochemical traits in pork (Kim et al., 2011), body composition traits (including carcass weight) (Kim et al., 2007), blood composition traits (Cho et al., 2011), as well as teat numbers and growth traits (Lee et al., 2003; Kim et al., 2005).

In this study, a GWAS was performed for $\mathrm{CW}$ in pigs derived from a $\mathrm{F}_{2}$ intercross between Landrace and $\mathrm{KNP}$ using high-density SNP chips to identify significant SNPs in candidate genes affecting the $\mathrm{CW}$ trait. These results will ultimately aid the design of breeding strategies for improving $\mathrm{CW}$ in pigs.

\section{MATERIALS AND METHODS}

\section{Animals and DNA extraction}

The pig population used in this study is the same previously described by Cho et al. (2011), which was the crossbred population between Landrace and KNP. This population was composed of 1,233 pigs from 79 full-sib $F_{2}$ families. The male animals in the $F_{2}$ population were not castrated. Genomic DNA extraction was carried out using $300 \mathrm{uL}$ of blood mixed with $900 \mathrm{uL}$ of red cell lysis solution $(0.8 \%$ ammonium chloride, $10 \mathrm{mM}$ Tris- $\mathrm{HCl}(\mathrm{pH} 7.2))$. DNA was subsequently extracted with modified methods from Miller et al. (1998) and RNase-treated (iNtRON, USA). DNA was collected using ethanol precipitation methods, diluted with TE buffer, and stored in refrigerators until their use.

\section{Genome-wide association study}

The CW was measured at the slaughterhouse. CW-related DNA markers were analyzed using 60K Porcine SNP chips (Illumina Inc., USA). The filtered SNPs for the analysis had a minor allele frequency less than $5 \%$, more than $10 \%$ of genotyping errors, less than or equal 0.001 for the Hardy-
Weinberg equilibrium error. At the end of filtration, 30,930 SNP markers remained for the GWAS. Following the genome-wide rapid association using a mixed model and regression (GRAMMAR) approach, a single SNP marker analysis was conducted to evaluate the association between $\mathrm{CW}$ and the SNP markers in this study population (Aulchenko et al. 2007). The CW trait was corrected for the fixed effect and random polygenic effects using a mixedffects model approach implemented in the ASREML program (VSN international, UK) according to the following model:

$$
\mathrm{Y}=\mu+\mathbf{s}+\mathbf{a}+\varepsilon-\operatorname{model} 1
$$

Here, $\mathrm{Y}=$ carcass weight phenotype, $\mu=$ overall mean, $\mathrm{s}=$ fixed effect of sex, $\mathrm{a}=$ random additive polygenic animal effect, $\varepsilon=$ random residual error.

The residuals derived from the mixed-effects model above were used as dependent variables in linear regression analyses to account for familial relatedness within the $F_{2}$ intercross population. The GWAS was performed under an additive model for each SNP using the PLINK program (Purcell et al., 2007) according to the following:

$$
\mathrm{Y}^{\prime}=\mathrm{b}_{0}+\mathrm{b}_{1} \text { Xsnp_a }+\varepsilon-\operatorname{model} 2
$$

Here, $\mathrm{Y}^{\prime}=$ residual obtained from model $1, \mathrm{~b}_{0}=$ intercept of regression equation, $b_{1}=$ regression coefficient of the additive effect of SNP markers, Xsnp_a= regression variable of the additive effect, $\varepsilon=$ random residual error.

The genome-wide threshold was applied using a Bonferroni correction (i.e., $0.05 /$ number of SNP markers; $P=1.61 \times 10^{-6}$ ).

\section{Positional candidate gene search and SNP geno- typing}

The positional candidate genes in the SSC12 CW QTL region were searched using the NCBI database (http://www. ncbi.nih.gov). The SNPs in the candidate genes were searched using previous results from the Hiseq 2000 (Illumina Inc., USA) database. Additional SNP genotyping was performed using the VeraCode GoldenGate assay (Illumina Inc., USA).

\section{Statistical analysis for positional candidate genes}


The phenotype was processed with the Minitab program (Minitab, PA, USA). The mixed-effects model (including sire and dam as the random effects as well as SNP genotype and sex as the fixed effects) was used to calculate the least squares mean and standard error of the SNP genotypes with the SAS program (SAS ver 9.1, SAS Institute Inc., NC, USA). The Plink program was used for the association analyses between SNP genotypes and CW.

\section{RESULTS \& DISCUSSION}

\section{Relationships of SNPs in the $60 \mathrm{~K}$ porcine chip with carcass weight}

Association tests were performed between carcass weight (CW) and $60 \mathrm{~K}$ SNPs (for pigs from the $F_{2}$ intercross population between Landrace and KNP), then visualized with the Manhattan plot. The results revealed two chromosomes, SSC5 and SSC12, with chromosomal regions with a genomewide significance level of 5.79 (Fig. 1). In particular, SSC12 contained nine statistically significant genes (Table 1).

Based on the database, 35 QTLs have been identified in most chromosomes, except chromosomes 11, 15, 16, 17, 18, and Y (Animal QTLdb, http://www.animalgenome.org/cgi-bin/ QTLdb/SS/index). Previous results indicated that six chromosomes (chromosome 3, 4, 5, 7, 8, 14) have significant associations with $\mathrm{CW}$ in White Duroc and Erhualian crosses (Ma et al., 2009). Similarly, Geldermann et al. (2010) suggested that seven chromosomes (chromosomes 1, 2, 4, 5, 7, 9, and $\mathrm{X}$ ) have significant associations with $\mathrm{CW}$ in Meishan and Pietrain crosses. None of the above publications found a significant association between $\mathrm{SSC} 12$ and $\mathrm{CW}$. However, Thomsen et al. (2004) found similar results in which SSC12 harbored a CW QTL in the $107.8 \mathrm{cM}$ region in Berkshire and Yorkshire crosses. Our results also demonstrated a significant association of $\mathrm{CW}$ with a region between the 88.5 and $113.1 \mathrm{cM}$ interval (SW1962-SWR1021). Therefore, significant SNPs in this region were further investigated to identify causative SNPs that directly affect $\mathrm{CW}$ in pigs.

\section{Identification of SNPs in significant QTL regions}

A total of nine candidate genes associated with $\mathrm{CW}$ were selected in the significant QTL region on SSC12 (LOC100523510, LOC100621652, LOC100521749, LOC100736982, MYHC, LOC100621981, DNAH2, MYOCD, WSB1). Among
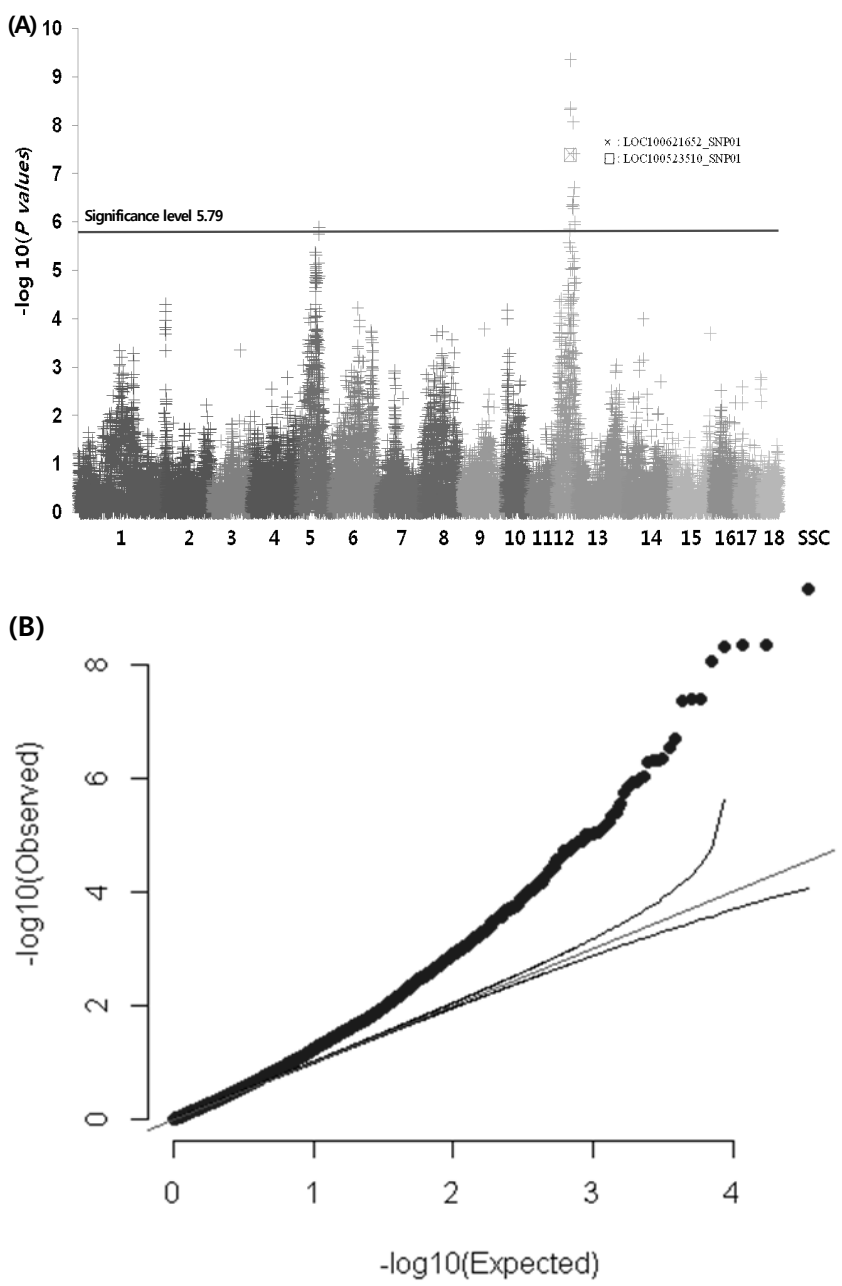

Fig. 1. The genome-wide association of SNPs with carcass weight (CW) (in a cross of Landrace and Korean native pigs) through 60K SNP analysis. (A) Association between CW and 30,917 mapped SNP markers in 18 pig autosomes using an additive model. On the $y$-axis are $\log 10$ ( $P$ values), and on the $x$-axis are the physical positions of the SNP markers by chromosome. The Bonferroni method cut-off value was 5.79 (i.e. $0.05 / 30,917=1.61 \times 10^{-6}$ ). (B) Quantile-quantile plots of SNP markers.

these, two significant candidate genes (LOC100523510, LOC 100621652) were further investigated because of their locations in the regions with the highest test statistics. The SNPs in the two positional candidate genes were investigated using previous genome sequencing information and five SNPs were identified. To validate the five SNPs from the two genes on SSC12, the VeraCode GoldenGate assay tool was applied. One SNP in the LOC100621652 gene and four SNPs in LOC100523510 gene were highly associated with the CW trait (Fig. 2). For the identified SNPs, the least 
Kang et al.; Genome Wide Association Analysis of Carcass Weight in Pig

Table 1. Summary of significant SNP markers on SSC12

\begin{tabular}{cccccc}
\hline SNP & CHR & Position & A1* & Nearest gene & $P$-value \\
\hline \hline ASGA0082658 & 12 & 49131683 & $\mathrm{C}$ & LOC100523510 & $4.50 \times 10^{-10}$ \\
ALGA0108818 & 12 & 49085493 & $\mathrm{~T}$ & LOC100621652 & $4.45 \times 10^{-9}$ \\
ASGA0101340 & 12 & 49202373 & $\mathrm{G}$ & LOC100523510 & $4.45 \times 10^{-9}$ \\
ALGA0108452 & 12 & 48842443 & $\mathrm{G}$ & LOC100521749 & $4.74 \times 10^{-9}$ \\
ALGA0067072 & 12 & 57831831 & $\mathrm{G}$ & LOC100736982 & $8.71 \times 10^{-9}$ \\
ASGA0055256 & 12 & 58105269 & $\mathrm{~T}$ & MYHC & $3.90 \times 10^{-8}$ \\
ASGA0055110 & 12 & 56362154 & $\mathrm{~A}$ & LOC100621981 & $4.31 \times 10^{-7}$ \\
M1GA0017062 & 12 & 57787203 & $\mathrm{C}$ & LOC100736982 & $4.73 \times 10^{-7}$ \\
ASGA0055081 & 12 & 55135676 & $\mathrm{G}$ & DNAH2 & $4.87 \times 10^{-7}$ \\
ASGA0096092 & 12 & 59699634 & $\mathrm{C}$ & MYOCD & $9.89 \times 10^{-7}$ \\
ASGA0096381 & 12 & 59752770 & $\mathrm{C}$ & MYOCD & $1.16 \times 10^{-6}$ \\
ALGA0122685 & 12 & 45749901 & $\mathrm{G}$ & WSB1 & $1.41 \times 10^{-6}$ \\
\hline
\end{tabular}

* Miner allele.
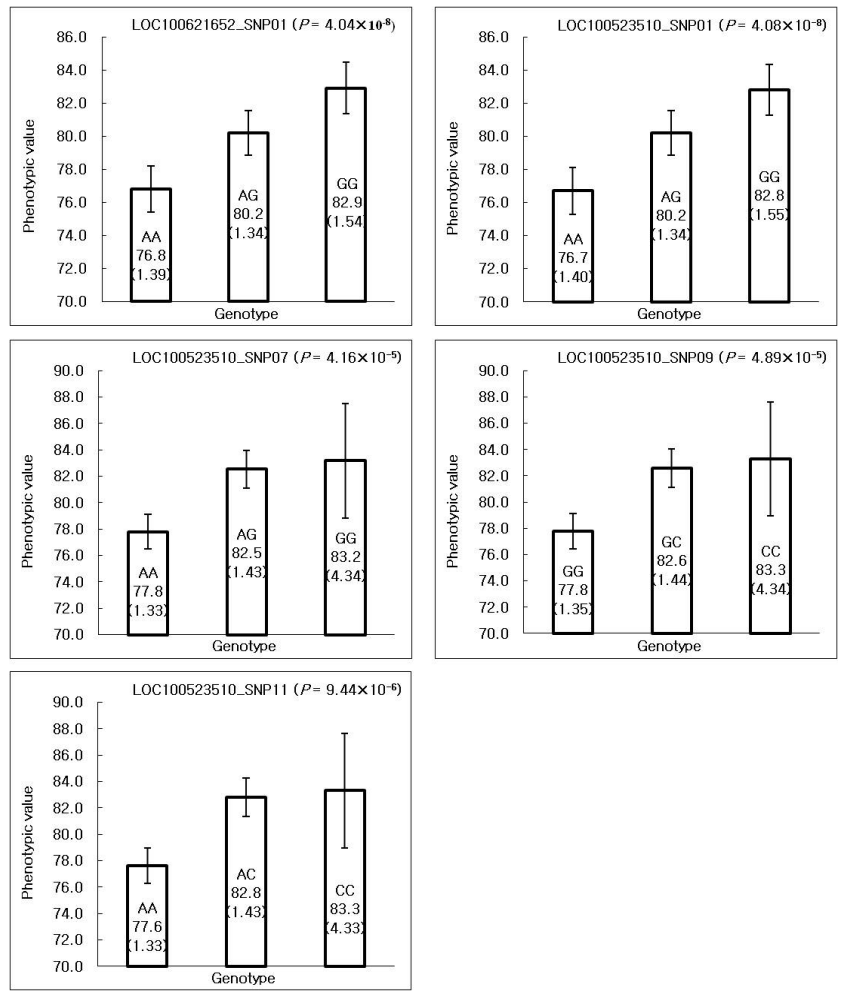

Fig. 2. The genotypic means and standard errors of carcass weight traits represent associations between the genotype of two candidate genes. $P$-values were computed based on the additive model using the Plink program. The units of the $y$-axis are in kilograms.

squares mean and standard error were calculated (Fig. 2). For SNP01 in the LOC100621652 gene, SNP01 in the
LOC100523510 gene and SNP07 in the LOC100523510 gene, the $\mathrm{G}$ alleles were associated with an increased $\mathrm{CW}$. However, the G allele of SNP09 in LOC100523510 and the A allele of SNP11 in LOC100523510 were associated with a decreased CW (Fig. 2).

Previously, Kim et al. (2007) constructed linkage maps using 133 microsatellite markers in an $F_{2}$ intercross between Korean native pig and Landrace from 318 pigs. This linkage analysis results indicated significant associations with $\mathrm{CW}$ in the $54 \mathrm{cM}(S W 1067)$ region on SSC6. Also, relationships with $\mathrm{CW}$ were observed in the $9 \mathrm{cM}(S W 983-S 0024)$ region on SSC9 and the $0 \mathrm{cM}(S W 2490)$ region on SSC12. However, these regions were not statistically significant in this population. In our results, a significant QTL on SSC12 was identified. Furthermore, five SNPs were validated that were directly associated with $\mathrm{CW}$. In terms of a QTL on SSC12 even though the same Korean native pig population was used for the QTL analysis, significant results were observed in different chromosomal regions. This indicates that statistical power and population differences may lead to different results.

In conclusion, a GWAS was performed for the $\mathrm{CW}$ trait in a $F_{2}$ intercross population between Landrace and $\mathrm{KNP}$. The results indicated that two genes, LOC100621652 and LOC100523510, on SSC12 show significant associations with the $\mathrm{CW}$ trait. The identification and further validation of five SNPs can potentially be applied to improve CW in Korean native pigs. 


\section{ACKNOWLEDGEMENTS}

This research was supported through the Technology Development Program (Project no. 110061031CG 000) of the Department of Agriculture and Forestry in the Ministry for Food, Agriculture, Forestry and Fisheries, Republic of Korea.

\section{REFERENCES}

Aulchenko, Y. S., de Koning, D. J. and Haley, C. 2007. Genomewide rapid association using mixed model and regression: a fast and simple method for genome-wide pedigree-based quantitative trait loci association analysis. Genetics 177, 577585.

Balding, D. J. 2006. A tutorial on statistical methods for population association studies. Nat. Rev. Genet. 7:781-791.

Cho, I. C., Park, H. B., Yoo, C. K., Lee, J. G., Lim, H. T., Lee, J. B., Jung, E. J., Ko, M. S., Lee, J. H. and Jeon, J. T. 2011. QTL analysis of white blood cell, platelet and red blood cell-related traits in an $\mathrm{F}_{2}$ intercross between Landrace and Korean native pigs. Anim. Genet. 42: 621-626.

Cho, S. H., Park, B. Y., Kim, J. H., Kim, M. J., Seong, P. N., Kim, Y. J., Kim, D. H. and Ahn, C. N. 2007. Carcass yields and meat quality by line weight of Korean native black pigs. Korean J. Anim. Sci. \& Technol. 49(4):523-530.

Cho, Y. M., Yoon, H. B., Lee, Y. C., Seo, K. S., Kim, S. D. and Park, Y. I. 2001. A study on growth characteristics of Korean native pig (KNP) and Landrace using individual growth curve parameters. J. Anim. Sci. \& Technol. 43(6):817-822.

Ernst, C. W. and Steibel, J. P. 2013. Molecular advances in QTL discovery and application in pig breeding. Trends Genet. 29(4):215-224.

Geldermann, H., Cepica, S., Stratil, A., Bartenschlager, H. and Preuss, S. 2010. Genome-wide mapping of quantitative trait loci for fatness, fat cell characteristics and fat metabolism in three porcine F2 crosses. Genet. Sel. Evol. 42:31.

Jeon, J. T., Park, E. W., Jeon, H. J., Kim, T. H., Lee, K. T. and Cheong, I. C. 2003. A large-insert porcine library with sevenfold genome coverage: a tool for positional cloning of candidate genes for major quantitative traits. Mol. Cells. 16(1): 113-116.

Jin, S. K., Kim, C. W., Song, Y. M., Jang, W. H., Kim, Y. B., Yeo, J. S., Kim, J. W. and Kang, K. H. 2001. Physicochemical characteristics of longissimus muscle between the Korean native pig and Landrace. Korean J. Food Sci. Ani. Resour. 21(2):142-148.

Kim, E. H., Choi, B. H., Kim, K. S., Lee, C. K., Cho, B. W.,
Kim, T. -H. and Kim, J. J. 2007. Detection of mendelian and parent-of-origin quantitative trait loci in a cross between Korean native pig and Landrace. I. Growth and body composition traits. Asian-Aust. J. Anim. Sci. 20(5):669-676.

Kim, S. W., Li, X. P., Lee, Y. M., Choi, Y. I., Cho, B. W., Choi, B. H., Kim, T. H., Kim, J. J. and Kim, K. S. 2011. QTL scan for meat quality traits using high-density SNP chip analysis in cross between Korean native pig and Yorkshire. Asian-Aust. J. Anim. Sci. 24:1184-1191.

Kim, T. H., Choi, B. H., Yoon, D., Park, E. W., Jeon, J. T., Han, J. Y., Oh, S. J. and Cheong, I. C. 2005. Identification of quantitative trait loci(QTL) affecting teat number in pigs. Asian-Aust. J. Anim. Sci. 17:1210-1213.

Kwon, O. S. 2006. Preservation background and environmental status of Korean native black pigs. Symposium proceedings of preservation and utilization of Korean native black pigs. pp. 3-20.

Lee, H. K., Lee, S. S., Kim, T. H., Jeon, G. J., Jung, H. W., Shin, Y. S., Han, J. Y., Choi, B. H. and Cheong I. C. 2003. Detection of imprinted quantitative trait loci (QTL) for growth traits in pigs. Asian-Aust. J. Anim. Sci. 16:1087-1092.

Ma, J., Ren, J., Guo, Y., Duan, Y., Ding, N., Zhou, L., Li, L., Yan, X., Yang, K., Huang, L., Song, Y., Xie, J., Milan, D. and Huang, L. 2009. Genome-wide identification of quantitative trait loci for carcass composition and meat quality in a large-scale White Duroc X Chinese Erhualian resource population. Anim. Genet. 40(5):637-647.

Miller, M. C., Sood, A., Spielvogel, B. F. and Hall, I. H. 1998. The synthesis and antitumor activity of the sodium salt and copper (II) complex of $\mathrm{N}$-[(trimethylamineboryl)-carbonyl]-Lphenylalanine methyl ester. Met. Based Drugs. 5:1-9.

Purcell, S., Neale, B., Todd-Brown, K., Thomas, L., Ferreira, M. A. R., Bender, D., Maller, J., Sklar, P., De Bakker, P. I. W., Daly, M. J. and Sham, P. C. 2007. PLINK: A tool set for whole-genome association and population-based linkage analyses. Am. J. Hum. Genet. 81:559-575.

Thomsen, H., Lee, H. K., Rothschild, M. F., Malek, M. and Dekkers, J. C. 2004. Characterization of quantitative trait loci for growth and meat quality in a cross between commercial breeds of swine. J. Anim. Sci. 82(8):2213-2218.

Yoo, C. K., Lim, H. T., Han, S. H., Lee, S. S., Ko, M. S., Kang, T., Lee, J. H., Park, H. B. and Cho, I. C. 2012. QTL analysis of back fat thickness and carcass $\mathrm{pH}$ in an $\mathrm{F}_{2}$ intercross between Landrace and Korean native pigs. Mol. Biol. Rep. 39:8327-8333.

(Received Jul. 24, 2013; Revised Aug. 19, 2013; Accepted Aug. 21, 2013) 